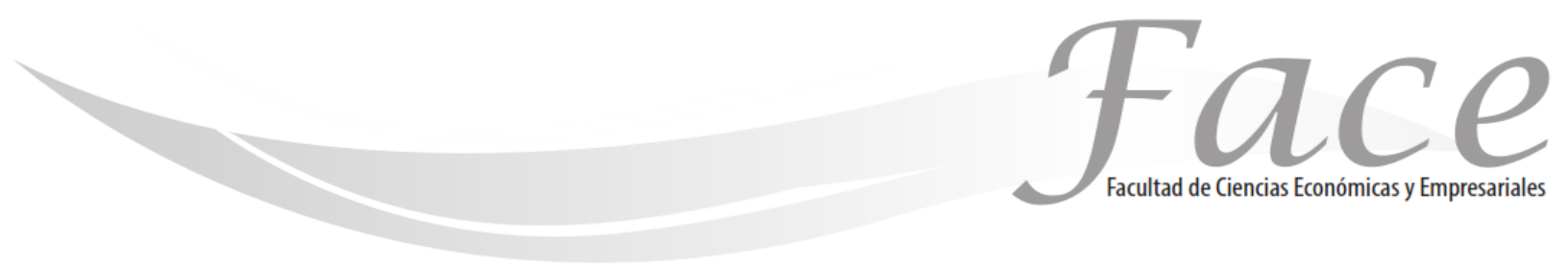

ISSN Impreso: 1794-9920

ISSN Electrónico: 2500-9338

Volumen $18-\mathrm{N}^{\circ} 2$

Año 2018

Págs. 58- 66

\title{
RELACIÓN ENTRE EL PRECIO DE LA ACCIÓN DE ECOPETROL Y EL VALOR DEL DIVIDENDO EN EL PERIODO 2008 AL 2017
}

\author{
Jorge Enrique Díaz-Pinzón * \\ Enlace ORCID: https://orcid.org/0000-0002-8870-7769
}

Fecha de Recepción: 28 de Junio 2018

Fecha de Aprobación: 22 de Octubre 2018

\section{Resumen:}

Este artículo analiza la correlación entre las variables dividendos y precio de la acción de Ecopetrol, y de esta manera dar una perspectiva a los accionistas de la empresa a generar oportunidades de crecimiento y de fomentar la mayor participación de inversionistas nacionales e internacionales.

Se realizó un estudio correlacional y lineal del precio de la acción de Ecopetrol y del valor de los dividendos entre el período 2008 al 2017. Para ello se utilizó la técnica econométrica financiera de la regresión lineal simple utilizando el programa SPSS v.20 con el fin establecer y describir la relación lineal entre dos variables cuantitativas continuas e independientes. Se observó que el p-valor de la prueba es igual a 0,005 , de esta manera se rechaza $\mathrm{Ho}$, es decir hay una correlación significativa entre el precio de la acción de Ecopetrol y el valor del dividendo durante el período 2008 a 2017. Se estableció que hubo una correlación significativa entre el precio de la acción de Ecopetrol y el valor del dividendo durante el período 2008 a 2017. Las ecuaciones lineales obtenidas fueron las siguientes: para el precio de cierre de Ecopetrol, $Y=153,98 X+312808, Y=-11,042 X+22380,679$ para el valor del dividendo.

Palabras clave: Análisis estadístico, análisis de regresión, dividendos, indicador económico, modelo matemático.

* Magíster en Gestión de la Tecnología Educativa, Docente titular de matemáticas e Investigador.

.Correo: jediazp@unal.edu.co, jorgediaz333@gmail.com 


\title{
RELATIONSHIP BETWEEN THE PRICE OF THE ECOPETROL ACTION AND THE VALUE OF THE DIVIDEND IN THE PERIOD 2008 TO 2017
}

\begin{abstract}
:
This article analyzes the correlation between the dividend variables and the share price of Ecopetrol, and in this way gives a perspective to the shareholders of the company to generate growth opportunities and to encourage the greater participation of national and international investors.

A correlation and linear study of Ecopetrol's share price and the value of the dividends between 2008 and 2017 was carried out. The financial econometric technique of the simple linear regression was used using the SPSS v.20 program in order to establish and describe the linear relationship between two continuous and independent quantitative variables. It was observed that the $\mathrm{p}$ value of the test is equal to 0.005 , in this way $\mathrm{Ho}$ is rejected, that is to say there is a significant correlation between the price of the Ecopetrol share and the value of the dividend during the period 2008 to 2017. It was established that there was a significant correlation between the Ecopetrol share price and the dividend value during the 2008 to 2017 period. The linear equations obtained were the following: for the Ecopetrol closing price, $Y=153.98 X+312808, Y=-11,042 X+22380,679$ for the dividend value.
\end{abstract}

Keywords: Statistical analysis; Regression analysis; Economic indicator; Mathematical model; Dividends.

\section{RELAÇÃO ENTRE O PREÇO DA ACÇÃO ECOPOROLÓGICA E O VALOR DO DIVIDENDO NO PERÍODO 2008 A 2017}

\section{Resumo:}

Este artigo analisa a correlação entre as variáveis de dividendos e o preço da ação da Ecopetrol e, dessa forma, dá uma perspectiva aos acionistas da empresa para gerar oportunidades de crescimento e incentivar a maior participação de investidores nacionais e internacionais.

Foi realizada uma correlação e estudo linear do preço da ação da Ecopetrol e do valor dos dividendos entre 2008 e 2017. A técnica econométrica financeira da regressão linear simples foi utilizada no programa SPSS v.20 para estabelecer e descrever a relação linear entre duas variáveis quantitativas contínuas e independentes. Observou-se que o p-valor do teste é igual a 0,005, desta forma Ho é rejeitado, ou seja, existe uma correlação significativa entre o preço da ação da Ecopetrol e o valor do dividendo durante o período de 2008 a 2017. Ficou estabelecido que havia uma correlação significativa entre a cotação da ação da Ecopetrol e o valor do dividendo durante o período de 2008 a 2017. As equações lineares obtidas foram as seguintes: para 0 preço de fechamento da Ecopetrol, $Y=153,98 X+312808, Y=-11,042 X+22380,679$ para o valor do dividendo.

Palavras-chave: Análise estatística; Análise de regressão; Indicador econômico; Modelo matemático; Dividendos 


\section{METODOLOGÍA:}

El trabajo de investigación se realizó mediante un tipo de investigación experimental, este tipo de investigación es:

Aquella que permite como mayor seguridad establecer relaciones de causa a efecto". Pues presenta una visión general y aproximada del objeto de estudio, además de contar una investigación de tipo cuantitativo. Se ha escogido una metodología de tipo cuantitativa, el diseño de la investigación cuantitativa establece un método experimental habitual del conjunto de las normas científicas. (Monje, 2011,p.105).

Para desarrollar la investigación se utilizó la base de datos del precio promedio por año de la acción de Ecopetrol, durante los años 2008 al 2017. (Ecopetrol, 2017b), además del valor del dividendo pagado a los accionistas en este mismo lapso de tiempo. Para ello se utilizó la técnica econométrica financiera de la regresión lineal simple utilizando el programa SPSS v.20, con el fin establecer y describir la relación lineal entre dos variables cuantitativas continuas e independientes.

Ecopetrol inició actividades en la cadena del petróleo como una Empresa Industrial y Comercial del Estado, delegada de administrar el recurso hidrocarburífero de la nación, y progresó en el orden en que otras concesiones restituyeron y concentró su operación. (Ecopetrol, 2014)

En 2003 el gobierno colombiano reformó la Empresa Colombiana de Petróleos, con el objetivo de internacionalizar y de esta manera hacerla más competitiva en el cuadro de la industria mundial de hidrocarburos. (Ecopetrol, 2014)

En el año 2007 la empresa de petróleos de Colombia Ecopetrol, ofreció acciones en una primera oferta 4.087.723.771 acciones, equivalentes a COP de \$ 5.722.813.279.400. El precio por suscripción fue de COP $\$ 1400$ por acción. (Ecopetrol, 2007)

A partir del año 2008 Ecopetrol realizó su primer pago de dividendos por un valor de COP \$ 115 por acción, en el año 2017 con pago de COP \$ 23. (Ecopetrol, 2017a)

El dividendo es la repartición del beneficio formado en la empresa entre todos sus accionistas. Banco Bilbao Vizcaya Argentaria (BBVA, 2009). El dividendo por acción es la cuantía de beneficio logrado por una empresa que se divide entre el número de acciones. (Economipedia, 2015)

Como interrogante de la investigación podríamos establecer que: ¿Existe algún tipo de correlación entre las variables, dividendos y el precio de la acción de Ecopetrol, en el período 2008 al $2017 ?$
El objetivo principal de las series de tiempo es realizar proyecciones o pronósticos sobre una actividad futura, admitiendo estables las condiciones y variaciones registradas hasta la fecha, lo cual admite planear y tomar disposiciones a corto o largo plazo. Después, con base en ese escenario ideal, que presume que los factores que influyeron en la serie en el pasado lo prolongarán haciendo en el futuro, se examinan las tendencias pasadas y el procedimiento de las actividades bajo la predominio de ellas; por ejemplo, en el predominio de ventas de un producto o de un servicio de una empresa se computan los posibles precios, la reacción del consumidor, el influjo de la competencia, etc. (Suárez, 2015)

El objetivo del análisis de regresión como método causal es predecir la demanda a partir de una 0 más principios (variables independientes), por ejemplo el tiempo, precios del producto o servicio, economía del país. (Ingenio empresa, 2017)

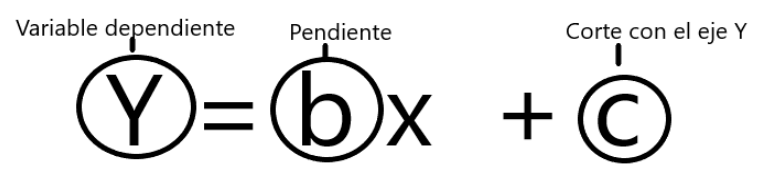


Figura 1. Ecuación de la recta. En ella:

La b es la pendiente de la recta.

La a es el punto de corte con el eje y.

La X es la variable independiente.

La Y es nuestra variable dependiente, precio de acción de Ecopetrol o del dividendo en un periodo.

Según (Valavanis, 1959), citado por (Paul, 2014). El objetivo de la econometría se puede atribuir a las teorías económicas bajo una forma matemática a fin de comprobarlas por métodos estadísticos y medir el impacto de una variable sobre otra, así como pronosticar económicamente ante resultados anhelados.

La econometría como un brazo de la economía que se identifica con los modelos matemáticos y estadísticos, de la programación lineal y la teoría de juegos; para estudiar, dilucidar y crear predicciones en la economía, vaticinando variables vitales para la misma como el precio, las reacciones del mercado, el coste de producción 0 las consecuencias de las políticas económicas. (Ruiz, 2015)

\section{RESULTADOS:}

\section{Hipótesis con el ANOVA}

La hipótesis del presente trabajo de investigación se diseña como una relación causal y se enuncia de la siguiente forma:

Hipótesis para las variables precio de la acción de Ecopetrol y precio del dividendo
Hipótesis Alterna (Ha): Correlacionan significativamente las variables precio de la acción de Ecopetrol y el precio del dividendo, durante el período 2008 al 2017.

Hipótesis Nula (Ho): No Correlacionan significativamente las variables precio de la acción de Ecopetrol y el precio del dividendo, durante el período 2008 al 2017.

\section{Prueba Estadística}

Se llevó a cabo una prueba de coeficiente de correlación de Pearson, que es un índice estadístico que evalúa la relación lineal entre dos variables cuantitativas. A diferencia de la covarianza, la correlación de Pearson es independiente de la escala de medida de las variables. (Parisca, 2009)

El valor del índice de correlación varía en el intervalo [-1, + 1].

Interpretación:

Si $r=0$ No existe correlación entre las variables Si $0.00 \leq r \pm 0.20$ existe correlación no significativa Si $\pm 0.20 \leq r< \pm 0.40$ existe una correlación baja Si $\pm 0.40 \leq r< \pm 0.70$ existe una correlación significativa Si $\pm 0.70 \leq r< \pm 1.00$ existe un alto grado de correlación Si $r=1$ existe una correlación perfecta positiva Si $r=-1$ existe una correlación perfecta negativa

En tabla 1, se aprecia el coeficiente de correlación de Pearson es de 0,802, es decir que se interpreta que existe un alto de grado de correlación entre las variables entre el precio de la acción de Ecopetrol y el valor del dividendo para el período 2008 al 2017.

En el análisis estadístico se incluirá lo siguiente:

El valor de significancia de la prueba es de a $=0.05(5 \%)$, si es > se acepta la hipótesis nula si es < se rechaza la hipótesis nula. Se utilizó el Software SPSS v. 20.0 .

En la tabla 1, se observa que el $p$-valor de la prueba es igual a 0,005, de esta manera se rechaza $\mathrm{Ho}$, es decir hay una correlación significativa entre el precio de la acción de Ecopetrol y el valor del dividendo durante el período 2008 a 2017.

\begin{tabular}{llccc}
\hline & \multicolumn{2}{c}{ Tabla 1. Correlaciones } & & \\
Año & Año & Dividendo & Precio Acción \\
& Correlación de Pearson & 1 &,- 313 &,- 350 \\
& Sig. (bilateral) & &, 378 &, 321 \\
Dividendo & $\mathrm{N}$ & 10 & 10 & 10 \\
& Correlación de Pearson &,- 313 & 1 &, $802^{* *}$ \\
& Sig. (bilateral) &, 378 & &, 005 \\
Precio- & $\mathrm{N}$ & 10 & 10 & 10 \\
Acción & Correlación de Pearson &,- 350 &, $802^{* *}$ & 1 \\
& Sig. (bilateral) &, 321 &, 005 & 10 \\
& $\mathrm{~N}$ & 10 & 10 & \\
\hline
\end{tabular}




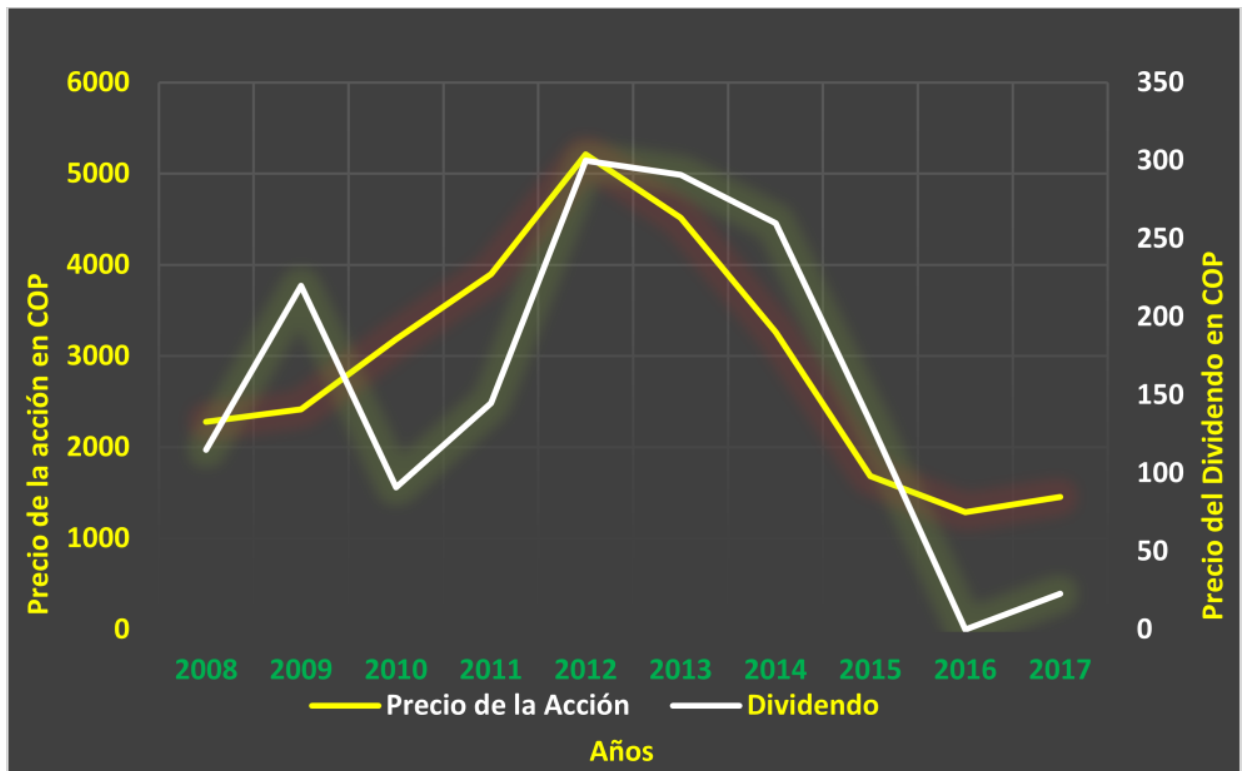

Figura 2. Precio de la acción de Ecopetrol respecto al precio del dividendo.

Fuente: Elaboración propia del autor

En la figura 2, se aprecia la tendencia del precio de la acción de Ecopetrol y el valor del dividendo, observamos que hay una relación entre las dos variables en el tiempo, al aumentar el precio de la acción, aumenta el valor del dividendo y viceversa. Se presentan unos puntos de corte entre el precio de la acción de Ecopetrol y el valor del dividendo, estos cortes se presentan en los años 2009, 2010, 2012 y 2016.

Tabla 2. Coeficientes-Precio de la acción de Ecopetrol

\section{Regresión Lineal}

Para realizar la regresión lineal, se trabajó con el promedio anual del precio de cierre de la acción de Ecopetrol durante el período 2008-2017 y el precio del dividendo. En la tabla 2, se observan los coeficientes para el precio de la acción de Ecopetrol y de allí obtener la ecuación lineal (1).

\begin{tabular}{|c|c|c|c|c|c|c|}
\hline & \multirow[t]{2}{*}{ Modelo } & \multicolumn{2}{|c|}{ Coeficientes no estandarizados } & \multirow{2}{*}{$\begin{array}{c}\begin{array}{c}\text { Coeficientes } \\
\text { tipificados }\end{array} \\
\text { Beta }\end{array}$} & \multirow[t]{2}{*}{$t$} & \multirow[t]{2}{*}{ Sig. } \\
\hline & & B & Error típ. & & & \\
\hline \multirow{2}{*}{1} & (Constante) & 312808 & 292953,308 & & 1,068 & $\overline{3,317}$ \\
\hline & Año & $-153,98$ & 145,567 &,- 350 & $-1,058$ &, 321 \\
\hline
\end{tabular}


Los coeficientes 153,98 y 312808 definen la recta. El coeficiente 153,98 es la pendiente de la recta; el cambio medio que se obtiene en el puntaje $(Y)$ por cada unidad de cambio que se produce de tiempo en minutos (X) le corresponde un decrimento de 312808 puntaje $(Y)$.

En la figura 3, podemos observar el diagrama de dispersión y la recta de regresión entre las variables tiempo en años (2008-2017) y el precio de la acción en este mismo período.
Los coeficientes $-11,042$ y 22380,679 definen la recta. El coeficiente $-11,042$ es la pendiente de la recta; el cambio medio que se obtiene en el puntaje (Y) por cada unidad de cambio que se produce de tiempo en minutos (X) le corresponde un decrimento de 22380,679 puntaje (Y). Ecuación (2).

$Y=-11,042 X+22380,679 \quad(2)$

En la figura 4, podemos observar el diagrama de dispersión y la recta de regresión entre las variables tiempo en años (2008-2017) y el valor del dividendo en este mismo período.

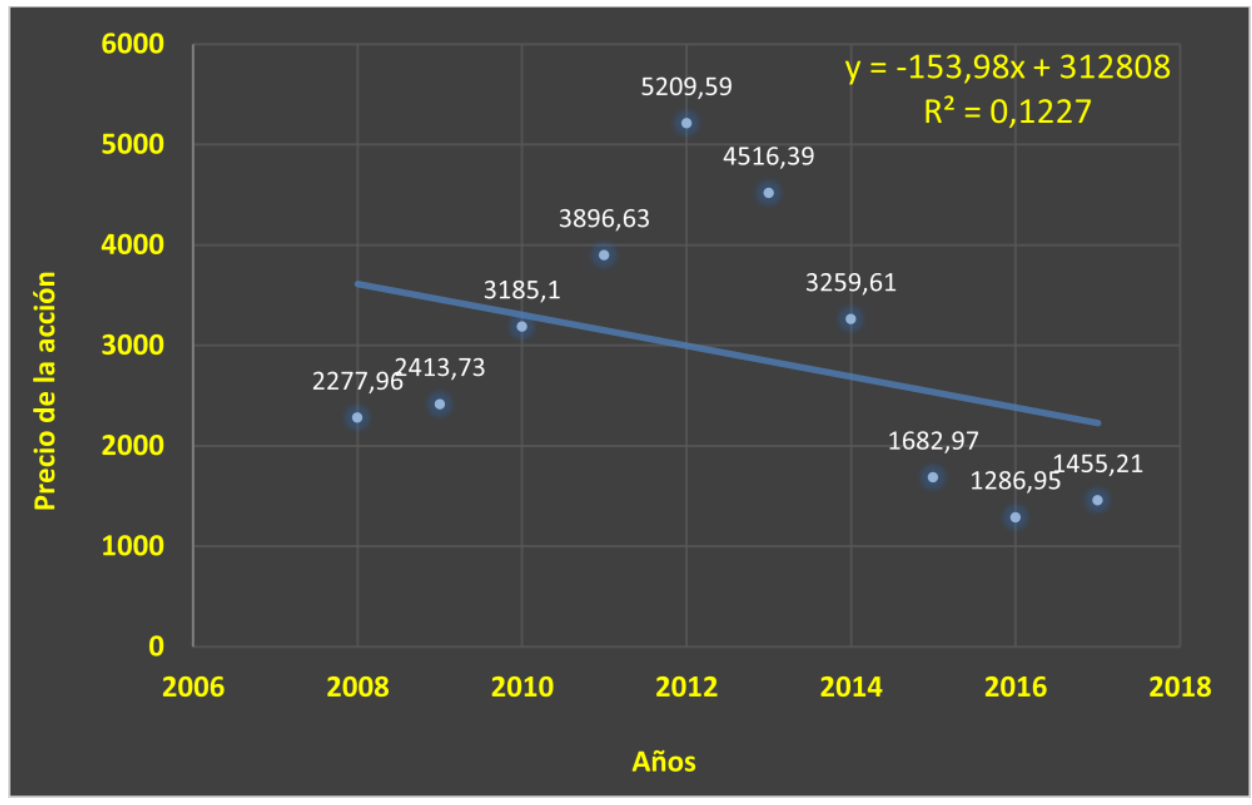

Figura 3. Diagrama de dispersión y recta de regresión.

Años-Acción de Ecopetrol

Fuente: Elaboración propia del autor

En la tabla 3, se observan los coeficientes para el precio del valor del dividendo y de allí obtener la ecuación lineal (2). 
Fuente: Elaboración propia del auto

\begin{tabular}{cc|c|c|c|c|c}
\hline \multirow{2}{*}{ Modelo } & \multicolumn{2}{|c|}{ Coeficientes no estandarizados } & $\begin{array}{c}\text { Coeficientes } \\
\text { tipificados }\end{array}$ & \multirow{2}{*}{ Sig. } \\
\cline { 3 - 5 } & $\mathrm{B}$ & Error típ. & Beta & & \\
\hline \multirow{2}{*}{1} & (Constante) & 22380,679 & 23807,342 & &, 940 &, 375 \\
& Año & $-11,042$ & 11,830 &,- 313 &,- 933 &, 378 \\
\hline
\end{tabular}

Los coeficientes $-11,042$ y 22380,679 definen la recta. El coeficiente $-11,042$ es la pendiente de la recta; el cambio medio que se obtiene en el puntaje (Y) por cada unidad de cambio que se produce de tiempo en minutos (X) le corresponde un decrimento de 22380,679 puntaje (Y). Ecuación (2).

\section{$Y=-11,042 X+22380,679 \quad(2)$}

En la figura 4, podemos observar el diagrama de dispersión y la recta de regresión entre las variables tiempo en años (2008-2017) y el valor del dividendo en este mismo período.

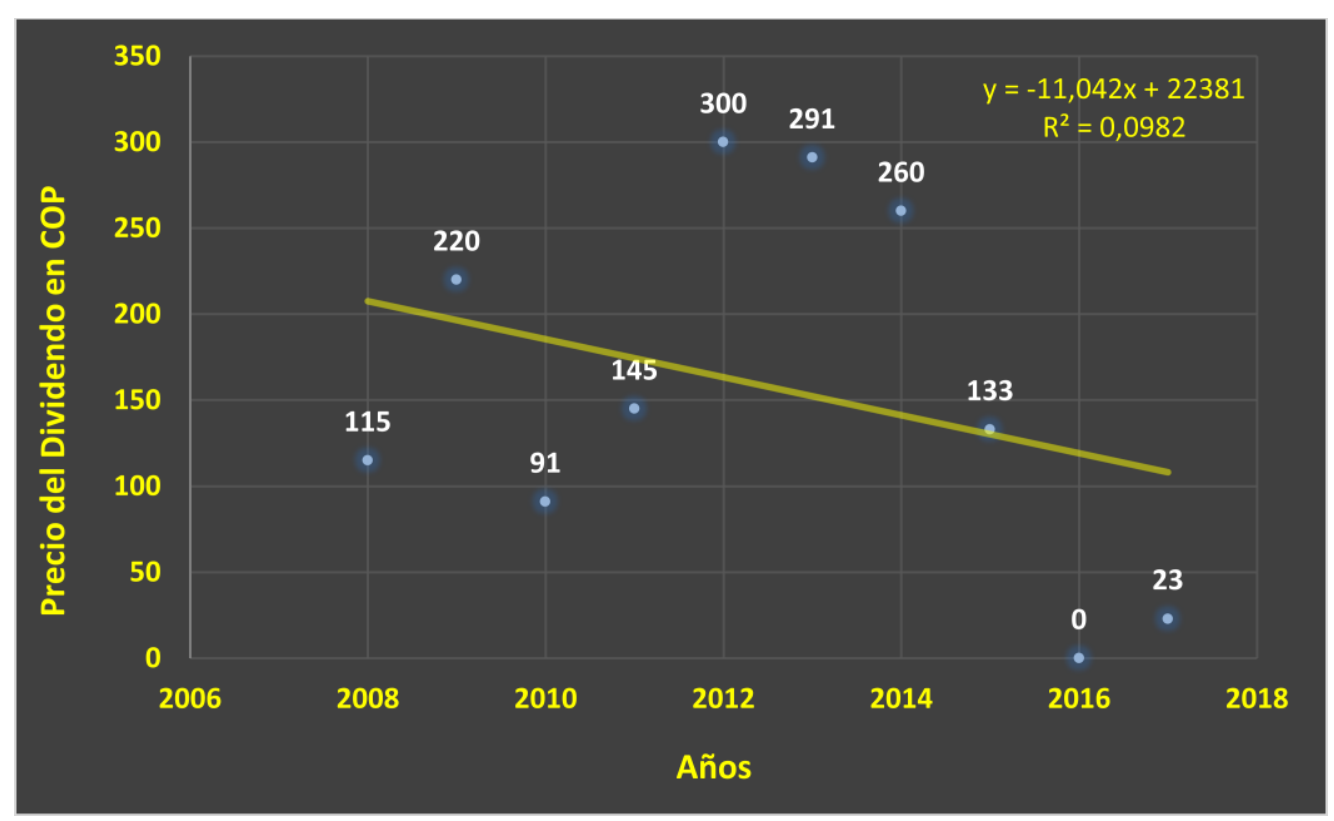

Figura 4. Diagrama de dispersión y recta de regresión.

Años-Valor del Dividendo 


\section{CONCLUSIONES:}

Se estableció según el objetivo de la investigación que por el coeficiente de correlación de Pearson fue de 0,802, esto nos indica que existe un alto de grado de correlación entre las variables entre el precio de la acción de Ecopetrol y el valor del dividendo para el período 2008 al 2017.

La evidencia obtenida sobre el período 2008 al 2017, indica que la caída del precio de la acción de Ecopetrol, también afecta de manera significativa el pago de dividendos a los accionistas de la empresa, como se analizó con los cálculos de regresión lineal.

Se observa un incremento positivo tanto del precio de la acción de Ecopetrol y el valor del dividendo del año 2008 hasta el 2012, de allí en adelante se aprecia decremento en estás dos variables, relacionadas con la baja del precio del petróleo Brent, hasta finales del año 2017.

Las ecuaciones lineales obtenidas fueron las siguientes: $Y=$ $-153,98 X+312808$ para el precio de cierre de Ecopetrol, , $Y=-11,042 X+22380,679$ para el valor del dividendo.

En el caso específico de los precios de la acción de Ecopetrol y los precios internacionales del petróleo, los análisis aplicados muestran una relación positiva fuerte entre las variables, los precios de la referencia Brent y WTI guardan una relación estrecha con el valor de la acción de Ecopetrol, por lo cual la propensión de una será similar en proporción en cuanto a variaciones de la otra.

Según (Cabestre,1196), menciona que, el pago de dividendos afecta, por termino medio, de forma positiva al comportameinto de precios de las acciones, cuya máxima mnisfestación se produce sobre la fecha ex dividendo.

Es posible elaborar modelos de pronóstico de activos financieros bajo el modelo de media móvil simple y media móvil exponencial esgrimiendo parámetros estadísticos para su evaluación, realizando simulaciones para los valores pronosticados a partir de los valores de series periódicas, en este caso series de años de las acciones de Ecopetrol y del dividendo particularidad del estudio. (Díaz, 2018)

Según (Alarcón, et al, 2007), se puede anhelar con un pronóstico es a esbozar el posible procedimiento del valor de una variable en el futuro, y por lo tanto disminuir esa incertidumbre. No hay que desconocer que todo pronóstico se hace bajo el aparente de que las condiciones actuales definitivas del valor de la variable en el pasado se conservan reinantes durante el periodo para el cual se efectúan las predicciones.

\section{REFERENCIAS:}

Alarcón, M. Rubio, H. Torres, R. Medina, J.Lozano, J.Estrada, R. (2007). Elección de un MODELO ECONOMÉTRICO DE PRONÓSTICO para el tipo de cambio en México. Recuperado el 19 de junio de 2018 de: http://www.uach.mx/extension_y_difusion/synthes is/2008/07/29/eleccion_de_un_modelo\%20econo metrico_de_pronostico_para_el_tipo_de_cambio_ en_mexico.pdf

Banco Bilbao Vizcaya Argentaria .BBVA (2009). Acciones y dividendos ¿cómo cambia su cotización con el pago de dividendos? Recuperado de: http://www.bbvacontuempresa.es/a/el-pago-dedividendos-afecta-a-la-cotizacion-de-las-acciones

Cabestre, F. (1996). La formación de precios de las acciones alrededor del pago de dividendos en el mercado de capitales Español. Revista Española de Financiación y Contabilidad. Vol. XXV. No. 86. Pp. 179-198.

Díaz, J. (2018). Análisis Econométrico de la acción de Ecopetrol. Editorial Académica Española. ISSN: 978-620-2-16002-5.

Economipedia (2015). Dividendo por acción. Recuperado de:

http://economipedia.com/definiciones/dividendopor-accion.html

ECOPETROL. (2007).Programa de emisión y colocación de acciones. Recuperado de: http://www.ecopetrol.com.co/wps/wcm/connect/es Id347784d-ec8a-4348-a5e4aa7efd60dd84/40386_CAPACITACION_ECOPET ROL_Primera_Ronda_DEFINITIVA.pdf?

ECOPETROL. (2014). Nuestra Historia. Recuperado de: https://www.ecopetrol.com.co/wps/portal/es/ecop etrol-web/nuestra-empresa/quienessomos/acerca-de-ecopetrol/nuestra-historia

ECOPETROL. (2017a). Histórico de Dividendos. Recuperado de: 
ISSN: 1794-9920 Julio - Diciembre de 2018

Volumen 18 Número 2, Año 2018 Págs. 58 - 66

https://www.ecopetrol.com.co/wps/portal/es/ecop etrol-web/relacioninversionistas/acciones/historico-dividendos

ECOPETROL. (2017b). Histórico de la acción. Recuperado de: http://www.ecopetrol.com.co/wps/portal/es/ecopet rol-web/historico-de-la-accion

Ingenio Empresa. (2017). La regresión lineal para pronosticar la demanda. Recuperado el: $18 \mathrm{de}$ junio de 2018 de: https://ingenioempresa.com/regresion-lineal/

Monje, C. (2011). Metodología de la investigación cuantitativa y cualitativa. Guía didáctica. Recuperado de: https:// carmonje.wikispaces.com/file/view/Monje+Carlos +Arturo+-+Gu\% $\%$ C3\% ADa+did\%C3\%A1ctica+Metodolog \%C3\%ADa+de+la+investigaci\%C3 \%B3n.pdf

Paul, J. (2014). Teoría y práctica de la econometría financiera. Volumen 1. Recuperado de febrero de 2018 de: https://repositorio.itesm.mx/ortec/bitstream/11285/ 622337/1/P221.pdf

Parisca, M. (2009). Coeficiente de la correlación de Pearson, media, moda y mediana. Recuperado el 19 de diciembre de 2017 de: http://html.rincondelvago.com/coeficientede-lacorrelacion-de-pearson-media-moda-ymediana.html

Ruiz, F. (2015). Qué es la Econometría. Recuperado el 19 de junio de 2018 de: https://www.educadictos.com/econometria/

Suárez, M. (2015). Series de tiempo con Excel. Recuperado el 20 de junio de 2018 de: http://www.monografias.com/trabajos-pdf5/seriestiempo-excel/series-tiempo-excel.shtml

Zerpa,K. Chaparro.E (2016). Políticas de dividendos. Recuperado el 5 de marzo de 2019 de: https://es.slideshare.net/KarinaZerpa2/trabajo-deinvestigacin-dividendos 